Editorial

\title{
Ongoing COVID-19 Global Crisis and Scientific Challenges
}

\author{
Iddya Karunasagar ${ }^{1}$ Indrani Karunasagar ${ }^{1}$ \\ ${ }^{1}$ Directorate of Research and Development, Nitte University, \\ Mangalore, Karnataka, India
}

J Health Allied Sci NU 2020;10:1-2

This volume of Journal of Allied Health Sciences (Nitte University) is coming out at a time when most countries in the world are at crossroads and looking toward science to deal with an unprecedented pandemic that has shaken the world economy. As on May 4, 2020, the disease caused by the novel coronavirus disease 2019 (COVID-19) has affected over 3.5 million people in over 200 countries with mortality close to 250,000. Detected as pneumonia of unknown cause in Wuhan, China, during late December 2019, the causative agent was quickly identified as a member of $\beta$-coronavirus and closely related to severe acute respiratory syndrome (SARS)-coronavirus, and hence designated SARS-CoV-2. The previous outbreak of SARS, which also originated in China in 2002 affected approximately 8,000 people in 26 countries during 2002 to 2003. But the experience of managing this epidemic did not seem to impact the management of COVID-19 as evidenced by the number of people affected and mortalities, particularly in Europe and North America. COVID-19 pandemic has raised so many scientific questions that still demand answers.

Though the causative agent was identified and its genetic makeup deciphered fairly quickly, the availability of rapid diagnostics has far exceeded the demand. The nucleic acid-based test reverse transcriptase real-time polymerase chain reaction (PCR) remains a gold-standard method. Nasopharyngeal swab (NPS) is the most common sample taken for testing but studies indicate that the viral load in upper respiratory tract may decrease as the disease progresses and effects lungs and NPS may yield negative results. Though SARS-CoV-2 affects mainly respiratory system and the transmission is thought to be mainly through respiratory droplets, the virus can affect number of human systems. Like SARS coronavirus, SARS-CoV-2 has been shown to use angiotensin converting enzyme (ACE2) as the receptor and this protein is abundant in lower respiratory tract. Cells in lung, intestine, kidney, and blood vessels express ACE2. SARS-CoV-2 has been detected in saliva with efficiency comparable to nasopharyngeal swab. ACE2 receptors have been found on host cells in tongue and salivary gland. This information would help testing laboratories since collection of salivary samples would be much easier compared with nasopharyngeal swabs.

Even enterocytes of small intestine contain ACE2 and a small percentage of COVID-19 patients may present only gastrointestinal symptoms. SARS-CoV-2 RNA has been detected in stool samples and also in sewage. There are unpublished reports from China that virus could be grown from stool samples indicating the presence of live virus. But currently data are lacking on the survival in stool and in aquatic environment. This information would be very important to understand the possibility of disease transmission through virus shed in the stool and fecal-oral route of transmission.

The transmission rate of SARS-CoV-2 has been found to be much higher than SARS-CoV. It has been hypothesized that though the two viruses share the same host cell receptor (ACE2), SARS-CoV-2 could be more infectious due to lower receptor binding domain (RBD)-ACE2 free energy and more flexible RBD of SARS-CoV-2. The spike (s) protein of SARS-CoV-2, which binds to the host receptor protein, has a furin-like cleavage site and could be cleaved by furin expressed in tongue cells.

The high disease number of infections in Europe and North America and very low number of cases in countries in South East Asia such as Vietnam, Lao, Cambodia, and Myanmar that share borders with China is rather intriguing. Several investigators have discussed the effect of temperature and humidity on transmission but significant numbers of cases reported from countries like Singapore make the interpretation of temperature and humidity on transmission difficult. Are there factors such as genetic susceptibility to this virus, any cross protection through prior exposure to other
Address for correspondence Iddya Karunasagar, PhD, Directorate of Research and Development, Nitte University, University Enclave, Medical Sciences Complex, Deralakatte, Mangalore 575018, Karnataka, India (e-mail: iddya.karunasagar@nitte.edu.in).
DOI https://doi.org/

10.1055/s-0040-1712785

ISSN 2582-4287.
Copyright (2020 Nitte University

(Deemed to be University)
License terms

$($ (1) $\Theta \circledast$ 
coronaviruses affecting the respiratory tract are questions that future investigations need to answer. Additionally, it is not well established whether SARS-CoV-2 transmission could occur through aerosols. SARS-CoV-2 has been detected in pets like cats. Though they may not cause symptoms, it is to be established whether domestic pets like cats can play a role in disease transmission. Understanding the transmission routes and mechanisms would greatly help development of measures to minimize transmission.

Currently, there are no established therapies for SARS-CoV-2, but there are trials on repurposing drugs that have been approved for human use for other conditions.
These include use of the malaria drug hydroxychloroquine, protease inhibitors like lopinavir/ritonavir, nucleotide analogues like remdesivir and others. Data on the benefits is still not conclusive, though some studies indicate promise. Use of convalescent plasma is also being tried in some countries and results of these studies are still awaited. The world is looking today for evidence coming from scientific studies and journals, like ours, that are keen to disseminate the results from scientific studies through open access publication.

\section{Conflict of Interest}

None declared. 